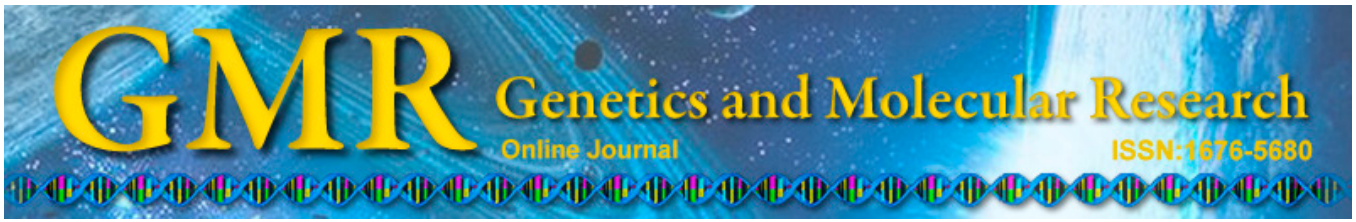

\title{
A single nucleotide polymorphism in the promoter region of let-7 family is associated with lung cancer risk in Chinese
}

\author{
L.Q. Shen ${ }^{1 *}$, Y.Z. Xie ${ }^{1 *}$, X.F. Qian ${ }^{2 *}$, Z.X. Zhuang ${ }^{1}$, Y. Jiao ${ }^{3}$ and X.F. Qi ${ }^{4,5}$ \\ ${ }^{1}$ Department of Oncology, The Second Affiliate Hospital of Soochow University, \\ Suzhou, China \\ ${ }^{2}$ Department of Experiment Diagnosis, \\ The First Affiliate Hospital of Soochow University, Suzhou, China \\ ${ }^{3}$ School of Radiation Medicine and Protection, \\ Medical College of Soochow University, Suzhou, China \\ ${ }^{4}$ Cyrus Tang Hematology Center, Soochow University, \\ Jiangsu Institute of Hematology, \\ The First Affiliated Hospital of Soochow University, Suzhou, China \\ ${ }^{5}$ Departments of Urology, The First Affiliated Hospital of Soochow University, \\ Suzhou, China \\ *These authors contributed equally to this study. \\ Corresponding author: X.F. Qi \\ E-mail: qixf-sz@hotmail.com
}

Genet. Mol. Res. 14 (2): 4505-4512 (2015)

Received July 1, 2014

Accepted October 29, 2014

Published May 4, 2015

DOI http://dx.doi.org/10.4238/2015.May.4.8

\begin{abstract}
Lung cancer is a complex polygenic disease and many genetic factors are involved in the development of the disease. As one of the most important and widely studied families of microRNA, let7 appears to play an important role in initiation and progression of lung cancer. Any small changes in miRNA level or its target point can cause significant changes in gene function. In this study, we examined whether a single-nucleotide polymorphism in the promoter region of the let-7 family (rs10877887) is associated with the susceptibility to
\end{abstract}


and prognosis of lung adenocarcinoma cancer. A hospital-based casecontrol research model was used in our study. The single-nucleotide polymorphism was genotyped in 69 lung cancer patients and 75 healthy controls by direct sequencing. The correlation between rs10877887 genotypes and the susceptibility to lung cancer was evaluated using an unconditional logistic regression model. Populations with the $\mathrm{CT}+\mathrm{CC}$ genotype had a significantly increased AC risk compared to those with the TT genotype $(\mathrm{CT}+\mathrm{CC}$ vs TT: $\mathrm{P}=0.043, \mathrm{OR}=2.032,95 \% \mathrm{CI}=$ 1.018-4.054). Furthermore, the risk effect was greater in subgroups of females over 60 years old $(\mathrm{CT}+\mathrm{CC} v s \mathrm{TT}$ : OR $=6.857,95 \% \mathrm{CI}=$ 1.425-33.008, $\mathrm{P}=0.012$ ), and the $\mathrm{C}$ allele were confirmed to be a risk factor related to lung cancer in these females $(\mathrm{P}=0.012)$. The singlenucleotide polymorphism rs10877887 in the promoter region of the let-7 family was found to be responsible for the susceptibility to lung adenocarcinoma cancer in Chinese individuals. This association was significantly stronger in females who were more than 60 years old.

Key words: Lung cancer; let-7; Single nucleotide polymorphism; Susceptibility

\section{INTRODUCTION}

Lung cancer is one of the most common malignant tumors worldwide and the morbidity and mortality continue to increase (Siegel et al., 2013). Because of the lack of effective methods for early diagnosis and treatment methods, the prognosis of lung cancer patients is typically very poor. The 5-year survival rate for non-small cell lung carcinoma (NSCLC) is only $17 \%$ (Chen et al., 2014). Lung cancer is a complex polygenetic disease that is often the comprehensive result of environmental influences and genetics (Shields, 2002). Additionally, lung cancer exhibits familial aggregation, indicating that hereditary susceptibility plays an important role in tumor occurrence and development.

MicroRNAs (miRNAs) are a class of endogenous, small (approximately $22 \mathrm{nt}$ ), noncoding, single-stranded RNAs that negatively regulate gene expression and function at the post-transcription level. An increasing number of studies have shown that miRNAs are involved in various developmental and physiological processes. Thousands of human genes or protein coding genes are microRNA targets (Xie et al., 2005; Lim et al., 2005; Lewis et al., 2005). let-7 was the first miRNA identified in humans; the hsa-let-7 family contains 13 members, including let- $7 \mathrm{a}-1 / 2 / 3$, let- $7 \mathrm{~b}$, let- $7 \mathrm{c}$, let- $7 \mathrm{~d}$, let- $7 \mathrm{e}$, let- $7 \mathrm{f}-1 / 2$, let- $7 \mathrm{~g}$, let- $7 \mathrm{i}$, mir- 98 , and mir-202 (Boyerinas et al., 2010). Furthermore, most of the family members were found to be downregulated in lung cancer and have been shown to act as either oncogenes or tumor suppressors involved in oncogenesis (Brueckner et al., 2007; Kumar et al., 2008; Raponi et al., 2009; Yang et al., 2010; Zhong et al., 2012; Zhan et al., 2013).

Single-nucleotide polymorphisms (SNPs) are important for increasing the diversity among individuals and influences phenotypes, traits, and diseases (Shastry, 2009). miRNArelated SNPs (miR-SNPs), particularly SNPs in miRNA genes and target sites, may influence miRNA expression and function, thus affecting phenotypes, cancer susceptibility, clinicopathological characteristics, therapeutic effects, or even patient survival time. Although a 
number of polymorphisms in let-7 binding sites have been reported to be involved in tumors, few studies have addressed the SNPs in the let-7 sequence, except for rs 10877887 , which is located in the promoter region of let-7i, and has been shown to be related to hepatocellular carcinoma (Xie et al., 2013). In this study, we examined SNP rs10877887 to determine its link with lung cancer.

\section{MATERIAL AND METHODS}

\section{Study subjects}

The case group consisted of 69 cancer patients that were randomly selected from the Second Affiliate Hospital of Soochow University between 2006 and 2009. All patients were pathologically diagnosed with lung adenocarcinoma after operation. The control group included 75 healthy subjects from the same hospital who had undergone physical examination during the same period. Furthermore, the control group was gender- and age-matched and had no blood relationships with any other subjects (Table 1). Samples were obtained after receiving consent from the participants or their family members. The genome refers to all genetic information regarding cells or viruses, and all cells in humans contain the same genetic information. Because lung tissue could not be obtained from healthy controls, we analyzed peripheral vein blood in these subjects.

\section{DNA extraction and genotyping}

Commercial kits, including the Wizard Genomic DNA Purification Kit (Promega, Madison, WI, USA) and the Formalin Fixation and Paraffin Embedded Tissue DNA Extraction Kit (Sangon Biotech, Shanghai, China), were used to extract genomic DNA from tumor tissues and peripheral blood, respectively (Lu et al., 2013). The primers for rs 10877887 were synthesized by Sangon Biotech according to the sequences in the literature (Huang et al., 2011) and had the following sequences: forward primer: 5'-TGGTGTCTGACT GCGC TTT-3'; reverse primer: 5'-CCGAGAGCTACGGGGATGA-3'. To amplify the DNA fragment, the following conditions were used: $95^{\circ} \mathrm{C}$ for $5 \mathrm{~min}$ for initial denaturation, 40 cycles at $95^{\circ} \mathrm{C}$ for $30 \mathrm{~s}, 60^{\circ} \mathrm{C}$ for $30 \mathrm{~s}$, and $72^{\circ} \mathrm{C}$ for $30 \mathrm{~s}$, followed by $72^{\circ} \mathrm{C}$ for $5 \mathrm{~min}$ for extension. The amplified products of rs 10877887 were subjected to direct DNA sequencing (BioSune, Shanghai, China).

\section{Statistical analysis}

Statistical analysis was performed using SAS version 17.0 (SAS Institute, Cary, NC, USA). All statistical tests were 2-sided, and differences were considered to be statistically significant when $\mathrm{P}<0.05$. Hardy-Weinberg equilibrium was analyzed using a goodness-of-fit $\chi^{2}$ test. To compare baseline characteristics, SNP genotypes, and allele frequency distributions in patients and healthy controls, we used the $t$-test for continuous variables and $\chi^{2}$ test for categorical data. Furthermore, odds ratios (ORs) and $95 \%$ confidence intervals $(95 \% \mathrm{CI})$ from a non-conditional logistic regression model, which had been adjusted by age and gender, were also used to estimate the association between rs10877887 genotypes and susceptibility to lung adenocarcinoma cancer. 


\section{RESULTS}

\section{Characteristics of cases and controls}

There were 144 Han Chinese ( 69 patients and 75 controls) included in this study. Their basic characteristics are shown in Table 1 . The case and control groups were age- $(\mathrm{P}=0.595)$ and sex-matched $(\mathrm{P}=0.430)$. Among the 69 lung cancer patients, $37(53.6 \%)$ had lymphatic metastasis, 10 (14.5\%) had high differentiation, 40 (58.0\%) had moderate differentiation, and $19(27.5 \%)$ had low differentiation. The proportion of TNM stage from I to IV was 37.7, 44.9, 14.5 , and $2.9 \%$, respectively. Further analyses demonstrated that lymph node metastasis and TNM stage affected patients' prognosis, and patients with lymphatic metastasis or those in an advanced TNM stage showed poorer prognosis (data not shown).

\begin{tabular}{|c|c|c|c|}
\hline \multirow[t]{2}{*}{ Variable } & Patients $(\mathrm{N}=69)$ & Controls $(\mathrm{N}=75)$ & \multirow[t]{2}{*}{$P$ value } \\
\hline & No. (\%) & No. (\%) & \\
\hline $\begin{array}{l}\text { Mean age (yeas) } \\
(\text { mean } \pm \text { SD) }\end{array}$ & $61.60 \pm 8.89$ & $61.28 \pm 8.08$ & $0.595^{*}$ \\
\hline Gender & & & $0.430^{* *}$ \\
\hline Male & $44(63.8)$ & $43(57.33)$ & \\
\hline Female & $25(36.2)$ & $32(42.67)$ & \\
\hline Age (years) & & & $0.776 * *$ \\
\hline$<60$ & $26(37.7)$ & $30(40.0)$ & \\
\hline$\geq 60$ & $43(62.3)$ & $45(60.0)$ & \\
\hline \multicolumn{4}{|l|}{ Differentiation } \\
\hline High & $10(14.5)$ & & \\
\hline Moderate & $40(58.0)$ & & \\
\hline Low & $19(27.5)$ & & \\
\hline \multicolumn{4}{|l|}{ TNM stage } \\
\hline I & $26(37.7)$ & & \\
\hline II & $31(44.9)$ & & \\
\hline III & $10(14.5)$ & & \\
\hline IV & $2(2.9)$ & & \\
\hline \multicolumn{4}{|c|}{ Lymphatic metastasis } \\
\hline Yes & $37(53.6)$ & & \\
\hline No & $32(46.4)$ & & \\
\hline
\end{tabular}

\section{Genotyping and association analysis}

Genotyping of SNP rs10877887 was successful in all study subjects by direct sequencing. The distribution of genotypes in patients and controls conformed to Hardy-Weinberg equilibrium ( $\mathrm{P}=0.112$ and 0.552 , respectively), and thus could be further analyzed.

As shown in Table 2, the frequency of the TT homozygote and CT $+\mathrm{CC}$ genotype were 28.99 and $71.01 \%$ in the patient group, while these values were 45.33 and $64.67 \%$ in the control group. After adjusting for age and gender by logistic regression analysis, we found significant differences in susceptibility to lung cancer by genotype (CT+CC vs TT: $\mathrm{P}=0.043$, $\mathrm{OR}=2.032,95 \% \mathrm{CI}=1.018-4.054)$. 
Table 2. Genotype distribution in cases and controls.

\begin{tabular}{lcccr}
\hline Genotypes & Cases $(\mathrm{N}=69)$ & Controls $(\mathrm{N}=75)$ & Odds ratio $(95 \% \mathrm{CI})$ & P value \\
\cline { 2 - 4 } & $\mathrm{N}(\%)$ & $\mathrm{N}(\%)$ & & \\
\hline Genotypes & & & & \\
TT & $20(28.99)$ & $34(45.33)$ & 1 (reference) & $\mathbf{0 . 0 4 3}$ \\
CT $+\mathrm{CC}$ & $49(71.01)$ & $41(64.67)$ & $\mathbf{2 . 0 3 2}(\mathbf{1 . 0 1 8 - 4 . 0 5 4 )}$ & $\mathbf{8}$ \\
\hline
\end{tabular}

Bold values are considered to be statistically significant.

Furthermore, when stratification analysis was carried out based on age and gender, similar results were obtained: the $\mathrm{CT}+\mathrm{CC}$ genotype significantly increased the risk of lung cancer $(\mathrm{CT}+\mathrm{CC}$ vs TT: $\mathrm{OR}=6.857,95 \% \mathrm{CI}=1.425-33.008, \mathrm{P}=0.012)$. The $\mathrm{C}$ allele may be a risk factor for lung adenocarcinoma cancer in females over 60 years of age (Table 3). No significant difference was observed in other subgroups, both in genotype distribution and overall survival of AC patients (data not shown).

\begin{tabular}{|c|c|c|c|c|}
\hline \multirow[t]{2}{*}{ Variables } & Cases $(\mathrm{N}=15)$ & Controls $(\mathrm{N}=19)$ & \multirow[t]{2}{*}{ Odds ratio $(95 \% \mathrm{CI})$} & \multirow[t]{2}{*}{ P value } \\
\hline & $\mathrm{N}(\%)$ & $\mathrm{N}(\%)$ & & \\
\hline \multicolumn{5}{|l|}{ Genotypes } \\
\hline TT & $3(20.00)$ & $12(63.16)$ & 1 (reference) & \\
\hline $\mathrm{CT}+\mathrm{CC}$ & $12(80.00)$ & $7(36.84)$ & $6.857(1.425-33.008)$ & 0.012 \\
\hline \multicolumn{5}{|l|}{ Alleles } \\
\hline $\mathrm{T}$ & $16(53.33)$ & $31(81.58)$ & 1 (reference) & \\
\hline $\mathrm{C}$ & $14(46.67)$ & $7(18.42)$ & $3.875(1.303-11.520)$ & 0.012 \\
\hline
\end{tabular}

Bold values are considered to be statistically significant.

\section{DISCUSSION}

Lung cancer is a complex polygenic disease. In addition to external environmental factors, numerous genetic factors such as changes in protein coding genes or non-coding genes can affect lung cancer development. miRNAs, a family of small noncoding RNAs, which are often reported to be up- or downregulated in lung cancer, appear to play a role in tumorigenesis. Moreover, many miRNAs are located in tumor-associated fragile sites, and any small changes in miRNA level or its target point can cause significant changes in cells. Although the mechanisms of the effect of miRNA on cancer are largely unknown, SNPs have been confirmed to be important. Some SNPs in pre-microRNAs, flanking regions, or target sites were shown to affect some physiological processes or were related to diseases.

Numerous miR-SNPs have been found to be closely associated with lung cancer. For example, rs11077, an SNP in the miRNA processing machinery genes of XPO-5, is involved in the chemotherapy response and survival of advanced NSCLC patients. Individuals carrying the $\mathrm{AC}$ genotype were generally more sensitive to chemotherapy and had longer survival times (Ding et al., 2013). The rs11077 AA genotype may be related to a higher recurrence rate in postsurgical NSCLC patients (Campayo et al., 2011). Among SNPs in pre-miRNAs, studies have reported that the variant homozygote CC of miR-196a2 rs11614913 was an important genotype that was significantly related to an increased risk and poorer survival of lung cancer (Hu et al., 2008; Tian et al., 2009). Similarly, Xu et al. (2013b) found that the G allele 
of rs895819 in pre-miR-27a was associated with shortened survival time of NSCLC patients. Additionally, the $\mathrm{AG} / \mathrm{GG}$ genotype exhibited a lower response rate to chemotherapy than the AA genotype (Xu et al., 2013b). In addition to these SNPs, polymorphisms in the binding site of miRNA also play an important role in lung cancer occurrence and survival. rs16917496 in SET8 3'-untranslated region (UTR), a binding site of miR-502, was related to cancer survival. The CC genotype was associated with longer survival time and a decreased risk of death in NSCLC patients (Xu et al., 2013a). Similarly, the CC+CT genotype was closely associated with longer survival of SCLC patients (Ding et al., 2012). SNP rs2239680 in the miR-335 binding site of the oncogene BIRC5 may alter the susceptibility to lung cancer. C allele carriers were found to be predisposed to lung cancer and advanced pathologic stage ( $\mathrm{Zu}$ et al., 2013).

let-7 is one of the most widely studied miRNAs and functions in many biological processes. Previous studies have reported that let-7 is downregulated in lung tumor tissues. Furthermore, lower expression is associated with a worse prognosis (Takamizawa et al., 2004; Yanaihara et al., 2006). Although the let-7 family has been widely examined, most previous studies have focused exclusively on the biological function and pathogenic role of let-7 itself, with few studies examining its gene polymorphisms.

To date, a number of studies have demonstrated that SNPs in let-7 binding sites are associated with carcinogenesis and survival. In an examination of the SNP in the 3'UTR of the KRAS gene located in the binding site of miRNA let-7, Chin et al. (2008) found that the variant allele at the LCS6 site led to increased KRAS expression and lower let-7 levels in lung tumor tissues compared to in adjacent tissues. Additionally, this variant was significantly associated with an increased risk for NSCLC among moderate smokers. Smits et al. (2011) demonstrated that this polymorphism may be a prognostic marker for colorectal cancer. Patients carrying the $\mathrm{G}$ allele live longer than those with the $\mathrm{T}$ allele. Researchers found that such functional SNPs are closely related to breast cancer risk (Paranjape et al., 2011), epithelial ovarian cancer risk (Pharoah et al., 2011), and prognosis of oral cancer (Christensen et al., 2009), among others.

In contrast to SNPs in 3'UTRs, few studies have examined the effect of SNPs in let-7 sequences. Two SNPs have been identified in the promoter region of let-7. Huang et al. (2011) found that both rs 13293512 of cluster let-7a-1/7f-1/7d and rs 10877887 of let-7i were unrelated to hepatocellular carcinoma susceptibility in a Chinese population. However, Xie et al. (2013) evaluated the associations between these 2 SNPs and survival of hepatocellular carcinoma patients and found that the $\mathrm{C}$ allele of rs 10877887 was significantly related to shorter survival. Inconsistent with the above results, we found in this study that rs 10877887 was related to the susceptibility of lung adenocarcinoma cancer but not to prognosis. Patients carrying the $\mathrm{CC}+\mathrm{CT}$ genotype were more likely to develop lung cancer than those carrying the TT genotype $(\mathrm{OR}=$ $2.032,95 \% \mathrm{CI}=1.018-4.054, \mathrm{P}=0.043)$. Moreover, the predictive effect became more prominent in females greater than 60 years of age $(\mathrm{P}=0.012, \mathrm{OR}=6.857,95 \% \mathrm{CI}=1.425-33.008)$, and the $\mathrm{C}$ allele may be a risk factor for $\mathrm{AC}$ in this subgroup $(\mathrm{P}=0.012)$.

This is the first study of polymorphisms in the promoter region of let-7 in lung cancer. However, there were some time and geographical limitations to our study. First, all subjects were selected from a single institution and the sample size was relatively small. Because selection bias may exist and the representativeness of samples may have been relatively weak, we can only draw preliminary conclusions. Second, the distributions of the rs 10877887 genotype may be very different between tumors, so the results obtained in our study may not agree with those of previous reports. Additional studies should examine the relationships between 
rs 10877887 and other pathological types of lung cancer or tumors. Third, only Chinese subjects were included in the study. Because allele sequence may vary in different ethnic groups, additional studies including different populations should be conducted.

In conclusion, although no significant prognostic value of rs 10877887 for lung cancer was identified in the present study, we found that the CT genotype and CT $+\mathrm{CC}$ genotype were significantly associated with an increased risk of lung adenocarcinoma. The $\mathrm{C}$ allele may be a risk factor for $\mathrm{AC}$ in females more than 60 years of age. Further investigations including larger populations are warranted to confirm our results and to define the potential mechanisms of rs10877887 in lung cancer.

\section{Conflicts of interest}

The authors declare no conflict of interest.

\section{ACKNOWLEDGMENTS}

Research supported partially by grants from the National Science Foundation of China (\#81170468 and \#81302382), the Natural Scientific Foundation of Jiangsu Scientific Bureau (\#BK2011266), the Priority Academic Program Development of Jiangsu Higher Education Institutions (PAPD), the High-level Talents in Six Industries of Jiangsu Province (\#WSN066), and the Pre-Research Project of the National Science Foundation of Soochow University (\#SDY2011B27).

\section{REFERENCES}

Boyerinas B, Park SM, Hau A, Murmann AE, et al. (2010). The role of let-7 in cell differentiation and cancer. Endocr. Relat. Cancer 17: F19-F36.

Brueckner B, Stresemann C, Kuner R, Mund C, et al. (2007). The human let-7a-3 locus contains an epigenetically regulated microRNA gene with oncogenic function. Cancer Res. 67: 1419-1423.

Campayo M, Navarro A, Viñolas N, Tejero R, et al. (2011). A dual role for KRT81: a miR-SNP associated with recurrence in non-small-cell lung cancer and a novel marker of squamous cell lung carcinoma. PLoS One 6: e22509.

Chen YB, Mu CY, Chen C, Huang JA, et al. (2014). Association between single nucleotide polymorphism of PD-L1 gene and non-small cell lung cancer susceptibility in a Chinese population. Asia Pac. J. Clin. Oncol. 10:e1-6.

Chin LJ, Ratner E, Leng S, Zhai R, et al. (2008). A SNP in a let-7 microRNA complementary site in the KRAS 3'-untranslated region increases non-small cell lung cancer risk. Cancer Res. 68: 8535-8540.

Christensen BC, Moyer BJ, Avissar M, Ouellet LG, et al. (2009). A let-7 microRNA-binding site polymorphism in the KRAS 3'UTR is associated with reduced survival in oral cancers. Carcinogenesis 30: 1003-1007.

Ding C, Li R, Peng J, Li S, et al. (2012). A polymorphism at the miR-502 binding site in the 3'-untranslated region of the SET8 gene is associated with the outcome of small-cell lung cancer. Exp. Ther. Med. 3: 689-692.

Ding C, Li C, Wang H, Li B, et al. (2013). A miR-SNP of the XPO5 gene is associated with advanced non-small-cell lung cancer. Onco. Targets Ther. 6: 877-881.

Hu Z, Chen J, Tian T, Zhou X, et al. (2008). Genetic variants of miRNA sequences and non-small cell lung cancer survival. J. Clin. Invest. 118: 2600-2608.

Huang F, Hu LM, Liu JB, Zhang YX, et al. (2011). Relationship between genetic polymorphism of promoter region let-7 and genetic susceptibility to hepatocellular carcinoma. Zhonghua Yu Fang. Yi Xue Za. Zhi. 45: 1093-1098.

Kumar MS, Erkeland SJ, Pester RE, Chen CY, et al. (2008). Suppression of non-small cell lung tumor development by the let-7 microRNA family. Proc. Natl. Acad. Sci. USA 105: 3903-3908.

Lewis BP, Burge CB and Bartel DP (2005). Conserved seed pairing, often flanked by adenosines, indicates that thousands of human genes are microRNA targets. Cell 120: 15-20.

Lim LP, Lau NC, Garrett-Engele P, Grimson A, et al. (2005). Microarray analysis shows that some microRNAs 
downregulate large numbers of target mRNAs. Nature 433: 769-773.

Lu Y, Lu J, Liu Q, Niu J, et al. (2013). A c.464 T>A mutation in VHL gene in a Chinese family with VHL syndrome. $J$. Neurooncol. 111: 313-318.

Paranjape T, Heneghan H, Lindner R, Keane FK, et al. (2011). A 3'-untranslated region KRAS variant and triple-negative breast cancer: a case-control and genetic analysis. Lancet Oncol. 12: 377-386.

Pharoah PD, Palmieri RT, Ramus SJ, Gayther SA, et al. (2011). The role of KRAS rs61764370 in invasive epithelial ovarian cancer: implications for clinical testing. Clin. Cancer Res. 17: 3742-3750.

Raponi M, Dossey L, Jatkoe T, Wu X, et al. (2009). MicroRNA classifiers for predicting prognosis of squamous cell lung cancer. Cancer Res. 69: 5776-5783.

Shastry BS (2009). SNPs: impact on gene function and phenotype. Methods Mol. Biol. 578: 3-22.

Shields PG (2002). Molecular epidemiology of smoking and lung cancer. Oncogene 21: 6870-6876.

Siegel R, Naishadham D and Jemal A (2013). Cancer statistics, 2013. CA Cancer J. Clin. 63: 11-30.

Smits KM, Paranjape T, Nallur S, Wouters KA, et al. (2011). A let-7 microRNA SNP in the KRAS 3'UTR is prognostic in early-stage colorectal cancer. Clin. Cancer Res. 17: 7723-7731.

Takamizawa J, Konishi H, Yanagisawa K, Tomida S, et al. (2004). Reduced expression of the let-7 microRNAs in human lung cancers in association with shortened postoperative survival. Cancer Res. 64: 3753-3756.

Tian T, Shu Y, Chen J, Hu Z, et al. (2009). A functional genetic variant in microRNA-196a2 is associated with increased susceptibility of lung cancer in Chinese. Cancer Epidemiol. Biomarkers Prev. 18: 1183-1187.

Xie K, Liu J, Zhu L, Liu Y, et al. (2013). A potentially functional polymorphism in the promoter region of let-7 family is associated with survival of hepatocellular carcinoma. Cancer Epidemiol. 37: 998-1002.

Xie X, Lu J, Kulbokas EJ, Golub TR, et al. (2005). Systematic discovery of regulatory motifs in human promoters and 3'UTRs by comparison of several mammals. Nature 434: 338-345.

Xu J, Yin Z, Gao W, Liu L, et al. (2013a). Genetic variation in a microRNA-502 minding site in SET8 gene confers clinical outcome of non-small cell lung cancer in a Chinese population. PLoS One 8: e77024.

Xu J, Yin Z, Shen H, Gao W, et al. (2013b). A genetic polymorphism in pre-miR-27a confers clinical outcome of nonsmall cell lung cancer in a Chinese population. PLoS One 8: e79135.

Yanaihara N, Caplen N, Bowman E, Seike M, et al. (2006). Unique microRNA molecular profiles in lung cancer diagnosis and prognosis. Cancer Cell 9: 189-198.

Yang Y, Li X, Yang Q, Wang X, et al. (2010). The role of microRNA in human lung squamous cell carcinoma. Cancer Genet. Cytogenet. 200:127-133.

Zhan M, Qu Q, Wang G, Liu YZ, et al. (2013). let-7c inhibits NSCLC cell proliferation by targeting HOXA1. Asian Pac. J. Cancer Prev. 14: 387-392.

Zhong Z, Dong Z, Yang L, Chen X, et al. (2012). Inhibition of proliferation of human lung cancer cells by green tea catechins is mediated by upregulation of let-7. Exp. Ther. Med. 4: 267-272.

Zu Y, Ban J, Xia Z, Wang J, et al. (2013). Genetic variation in a miR-335 binding site in BIRC5 alters susceptibility to lung cancer in Chinese Han populations. Biochem. Biophys. Res. Commun. 430: 529-534. 